Journal of Nature, Life

and Applied Sciences

Volume (5), Issue (4) : 30 Dec 2021

P: $47-62$

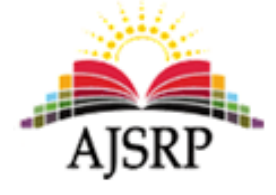

ISSN: 2522-3356
مجلة العلوم الطبيعية

والحياتية والتطبيقية

المجلد (5) ، العدد (4) : 30 ديسمبر 2021م

ص: 47 - 62

\title{
Gravitational Energy Levels: Part Two
}

\section{Edward S. Tannous}

Abstract: We present here a model that explains in a simple, easy and summarized manner, the values, meaning and reasons for the force of gravity, using simple physical tools. According to this model, a gravitational field actually creates different energy levels, similar to the atom, around the center of mass of the gravitational source, and a transition between the energy levels results in the creation of the force of weight acting on each small body which is in the gravitational field. As the body approaches a gravitational field, its energy value decreases to a value of $m_{0} u^{2}(R)$, proportional to the distance $R$ between the centers of the masses, when $u_{(R)}$ is the magnitude of the self-speed of light vector (the progression in the time axis) of the small body, and its value decreases as it approaches the center of the origin of the field. This change in the energy levels is the cause of the force of gravity. A formula is obtained for the concept of potential gravitational energy and the variables on which it depends, and for the time differences between two frames that are in the gravitational field, taking into account the motion and location of each frame. It is obtained from this model that the speed of light is also a variable value as a result of the effect of the gravitational field.

Keywords: Force of gravity, potential energy, kinetic energy, time differences between frames, gravitational curvature of light beams.

\section{مستويات طاقة الجاذبية: الجزء الثاني}

\author{
أدوارصليبا طنوس
}

المستخلص: يتلخص هذا البحث، بمعالجة النظرية النسبية العامة، من منظور مختلف عما طرحاه العالم ألبرت أينشتاين، وذلك طبقالقوانين

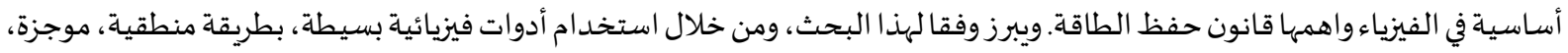
سهلة ومختصرة، نموذج فيزيائي خاص، يشرح طبقا له، القيم الدقيقة والمعنى الحقيقي وأسباب قوة الجاذبياة بين وفين الكتل.

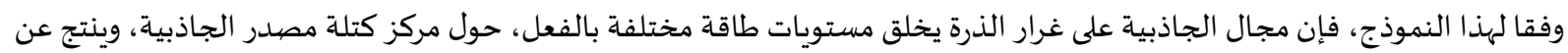

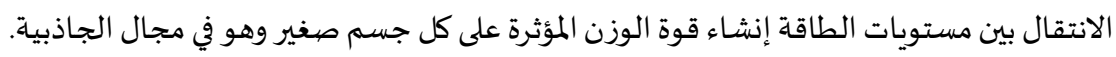

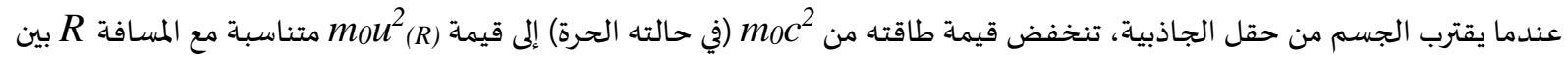
مراكز الكتلتين، حيث تنخفض قيمة مقدار المتجهاه لسرعة الضوء الذاتي (R) (السرعة في المحور الزمني) للجسم الصغير، كلما اقترب من مركز أصل المجال. مع الأخذ في الاعتبار حركة كل إطار ومن خلالها، يتم الحصول على معادلة دقيقة لمفهوم الطاقة الكامنة (طاقة الوضيع) والمتغيرات التي تعتمد

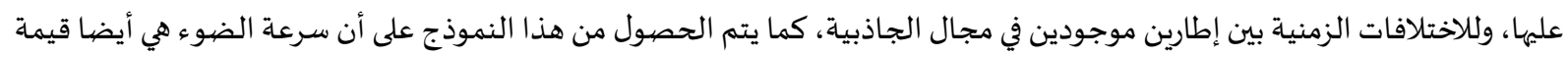
متغيرة نتيجة لتأثير حقل الجاذبية.

الكلمات المفتاحية: قوة الجاذبية، الطاقة الكامنة، الطاقة الحركية، الفروق الزمنية بين الإطارات، الانحراف الضوئي الثقالي. 
تعتبر هذه المقالة استمرارا للمقالات السابقة المعنونة تحت اسم "الكتلة السلبية" كما هو موضح في المرجع باللغة

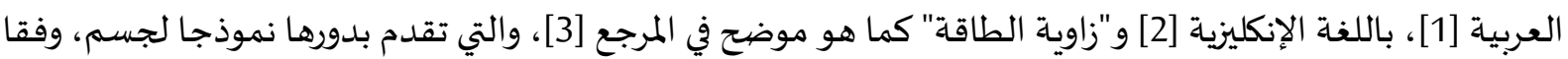

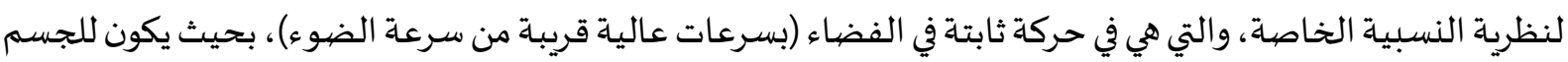
سرعة مساوية لسرعة الضوء في الزمكان، ولها زاوية طاقة وكتلة سالبة. يوضح هذا النموذج أن كل جسم يتحرك بسرعة الضوء في الزمكان، وفي اتجاه مختلف، وهو ولفه ما يسمى " المتجهاه

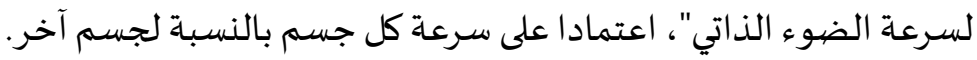

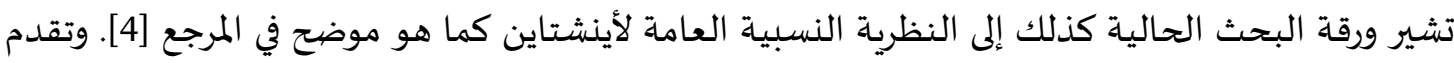

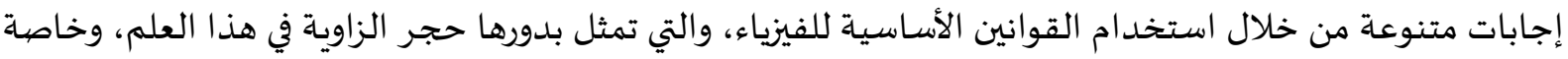

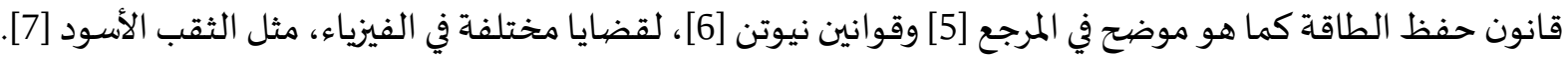

تعطى صيغة ألبرت أينشتاين التي تصف الطاقة الباقية للجسم بكتلة m بواسطة الصيغة

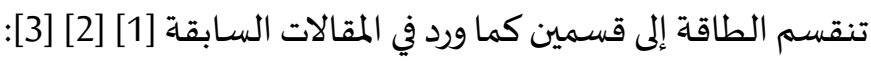

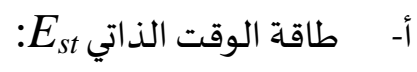
والتي تحدد مقدار الطاقة المتبقية في الكتلة نتيجة السرعة.

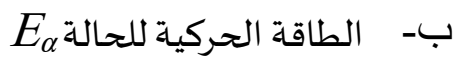

هي الطاقة التي يحملها الجسم بداخله في الإطار المرجعي نتيجة السرعة.

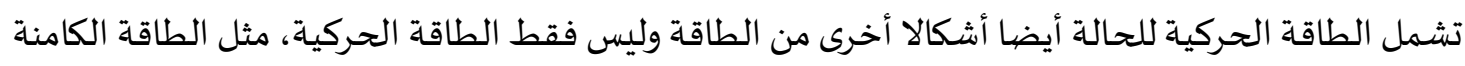
(الوضع). لذلك فإن الطاقة الكلية للجسم هي: E= E م=E $=E_{0}=E_{s t}+E_{\alpha}=m_{0} c^{2}$ من

1- - جسم صغير في مجال جاذبية، مصدرها كتلة كبيرة نشير في البداية إلى جسم صغير يقترب من جسم كبير تحت تأثير مجال الجاذبية. يفقد الجسم بعضيا من طاقة وقته الذاتية حجم الطاقة التي يفقدها يساوي مقدار العمل المستثمر وهو: الجسم الصغير m ومركز الجسم الكبير M، كما هو موضح في الشكل 1 (أ): 


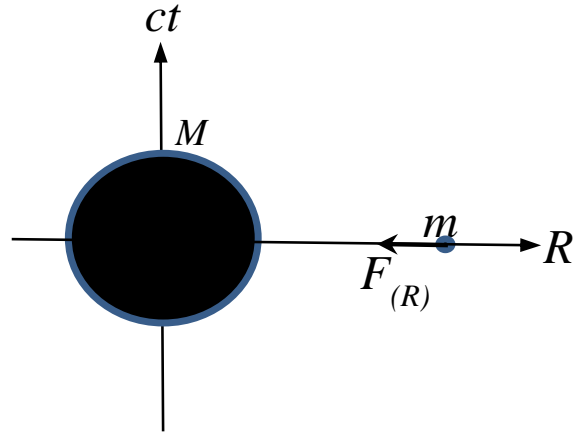

(ا)

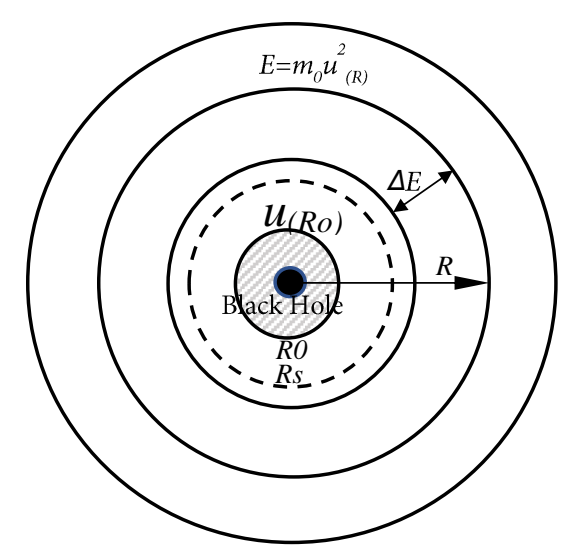

(ب)

الشكل (1) (أ): جسم صغيرm تحت تأثير مجال الجاذبية لجسم كبيرM.

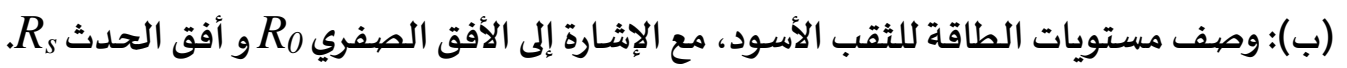

طبقا لذلك، فإن طاقة الوقت الذاتي (طاقة الكتلة)

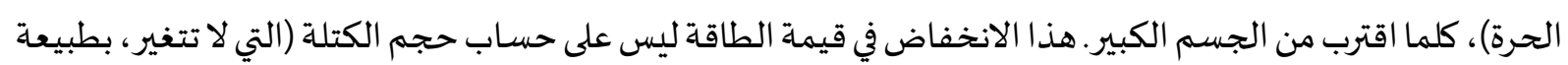

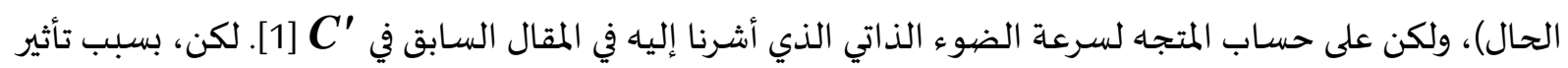

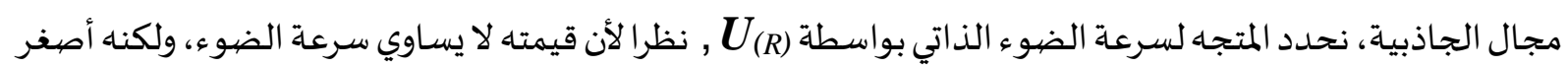

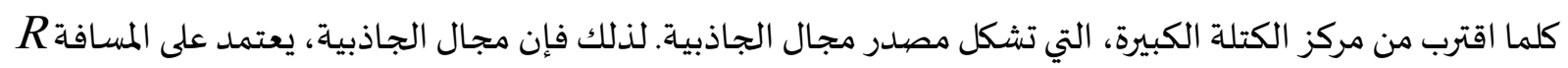
(البعد بين مركز الكتلتين)، بالتالي نعرف قيمته المطلقة:

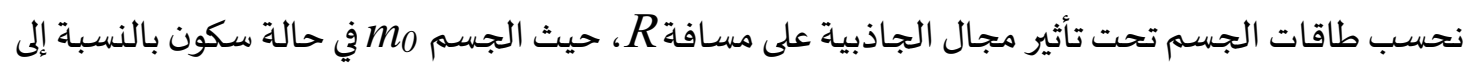
M أي ان زاوية الطاقة 0 =0 كما هو موضيح في الشكل 2 (أ): طاقة الوقت الذاتي (طاقة الكتلة): [1] $E_{s t}=m_{0} \boldsymbol{C} \boldsymbol{U}_{(R)}=m_{0} c u_{(R)}$

$$
\begin{aligned}
& \text { ( المتجها لسرعة الضوء الذاتي للجسم الكبير. }
\end{aligned}
$$

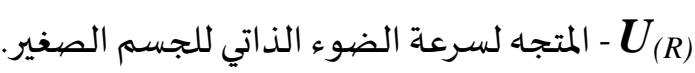

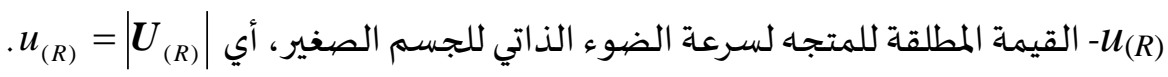




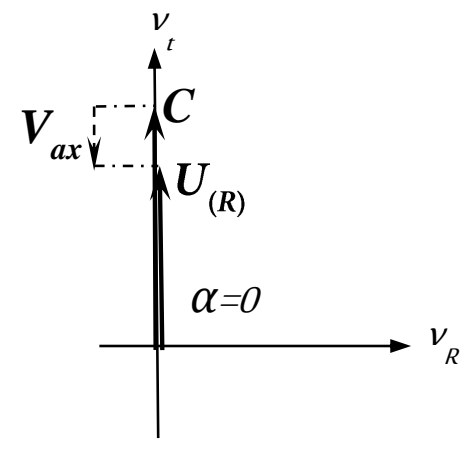

(i)

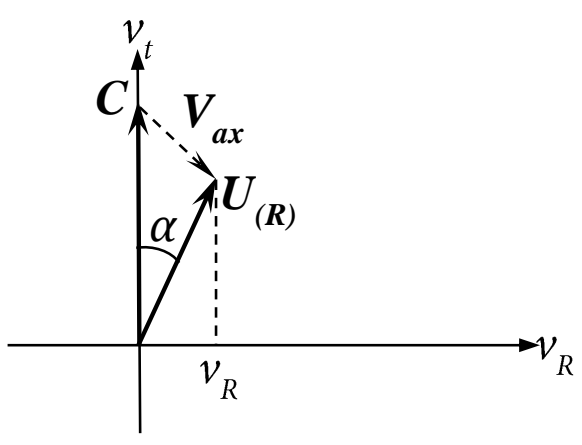

(ب)

الشكل (2) (أ) تبيان المتجده لسرعة الضوء الذاتي للجسم الكبيرC والجسم الصغير(R) (U) في حالة السكون.

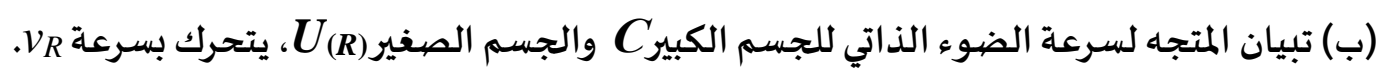

الطاقة الحركية للحالة، وهي الطاقة الكامنة (الوضع) فقط في هذه الحالة، حيث لا توجد حركة في الاتجاه R: [2] $E_{\alpha}=m_{0} \boldsymbol{U}_{(R)} \underbrace{\left.\boldsymbol{U}_{(R)}-\boldsymbol{C}\right)}_{\boldsymbol{V}_{a x}}=m_{0} u_{(R)}^{2}-m_{0} c u_{(R)}$

لذلك، فإن الطاقة الإجمالية للجسم الصغير تحت تأثير مجال الجاذبية هي:

[3] $E=E_{s t}+E_{\alpha}=m_{0} u^{2}(R)$

في المرحلة الأولية، إذا كان جسم صغير $m_{0}$ في حالة سكون على مسافة R من جسم كبير M.

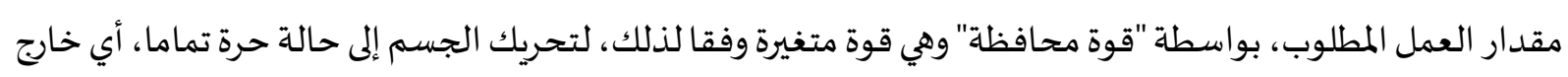
تأثير الجاذبية، باستخدام قانون نيوتن للجاذبية:

[4] $W_{(R \rightarrow \infty)}=\int_{R}^{\infty} F_{(R)} \mathrm{d} R=\int_{R}^{\infty} m_{0} \frac{G M}{R^{2}} \mathrm{~d} R=m_{0} \frac{G M}{R}$

$$
\text { حيث Gو هو ثابت الجاذبية لنيوتن. }
$$

وفقا لقانون حفظ الطاقة، فإن مقدار العمل الذي حسبناه في الصيغة 4 هو نفس كمية الطاقة التي يفقدها الجسم بإقتربه في مجال الجاذبية لمسافة R، أي، يفقده الجسم من طاقة الوقت الذاتي (طاقة الكتلة). من خلال استخدام الصيغة 1، نحصل على الفرق بين طاقة الوقت الذاتي (طاقة الكتلة) في حالة حرة تماما

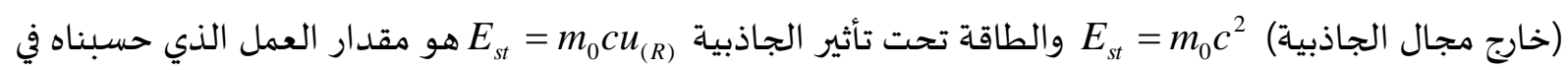

[5] $\Delta E_{s t}=m_{0} c^{2}-m_{0} c u_{(R)}=W_{(R \rightarrow \infty)}=m_{0} \frac{G M}{R}$ 
نتحصل على مقدار قيمة المتجه لسرعة الضيوء الذاتي لكل جسم صغير، والذي يقع على مسافة R من جسم

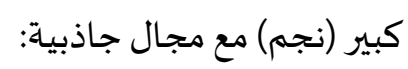

[6] $\quad u_{(R)}=c-\frac{G M}{c R}$

Uا $u_{(R)}$ يكون أصغر من سرعة الضوءc في قيمته المطلقة.

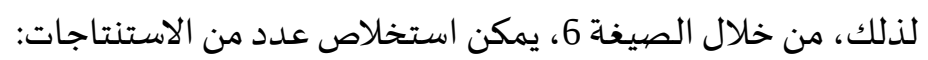

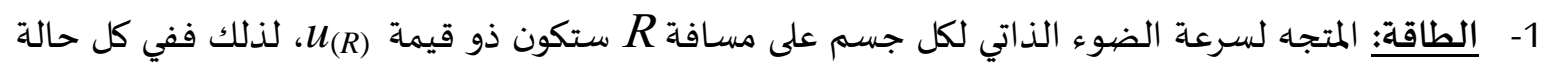
وجودية سيكون إجمالي الطاقة لديها الحركية للحالة طاقته وفقا لذلك.

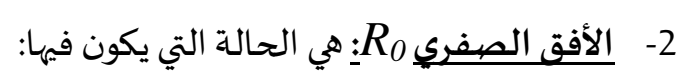

[7] $\quad R_{0}=\frac{G M}{c^{2}}$

من خلال الصيغة 6، يبدو أن المتجهه لسرعة الضوء الذاتي للجسم في هذه الحالة ستكون مساوية للصفر، أي

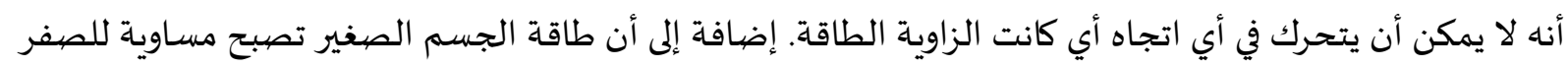
E= E $=E_{s t}=E_{\alpha}=0$

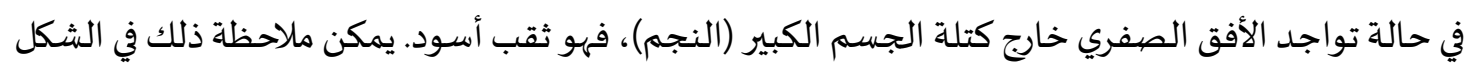

3- الوقت: كل جسم في حالة راحة، على مسافة R، سيكون له وقت أبطأ من الجسم الحر بفارق

$$
\Delta T=\frac{c T-u_{(R)} T}{c}=\frac{G M}{c^{2} R} T=\frac{R_{0}}{R} T
$$

4- الكتلة: على الرغم من أن الكتلة $m_{0}$ لا تتغير في الشكل والتركيب، إلا أن قيمتها المقاسة m (الكتلة الفعالة) صغيرة

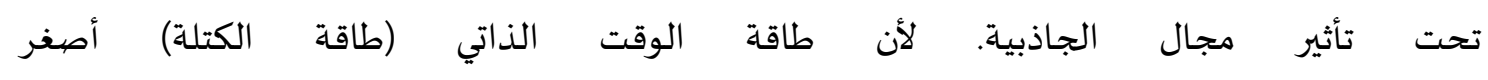

$$
E_{s t}=m_{0} \boldsymbol{C} \boldsymbol{U}(R)=m_{0} c u_{(R)} \cos \alpha=m c^{2}
$$

(x) هي الزاوية الطاقة، بافتراض أن الجسم يتحرك في اتجاه ما في الفضاء الإقليدي (x,y,z)، بحيث تكون سرعته

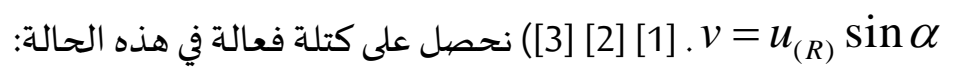

[8] $\quad m=m_{0} \frac{u_{(R)}}{c} \cos \alpha=m_{0}\left(1-\frac{G M}{c^{2} R}\right) \cos \alpha=m_{0}\left(1-\frac{R_{0}}{R}\right) \cos \alpha$

5- قوة الجاذبية: [9] يتم إنشاؤها في الواقع نتيجة للانتقال بين مستويات الطاقة المختلفة، انظر الشكل 1 (ب).

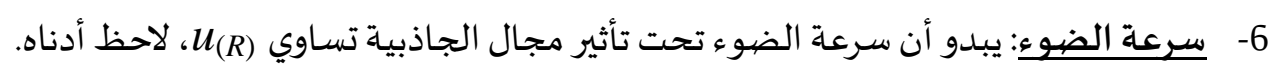


فشل العديد من الفيزيائيين في تفسير حقيقة أن الطاقة الكامنة E

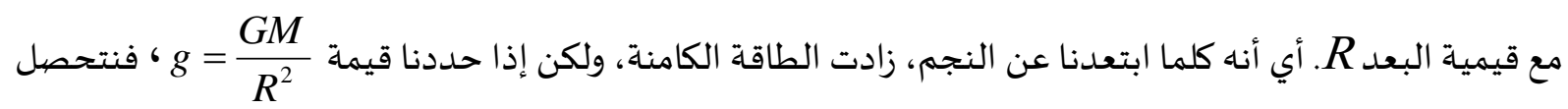
بذلك: بالإضافة إلى ذلك، هناك سؤال آخر يتمثل، فيما إذا كانت الطاقة الكامنة موجبة أم سلبية. لذا سوف نقدم هنا تعبيرا دقيقا عن طاقة الكامنة (الوضع) للجاذبية.

الطاقة الحركية للحالة Eم هي الطاقة التي تحتوي بداخلها على طاقتين، كل من الطاقة الحركية والكامنة. إذا

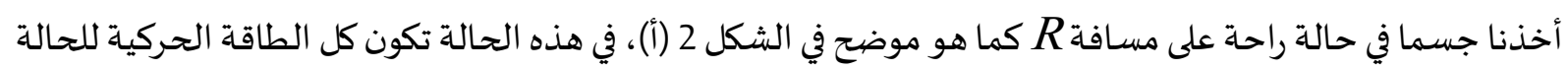

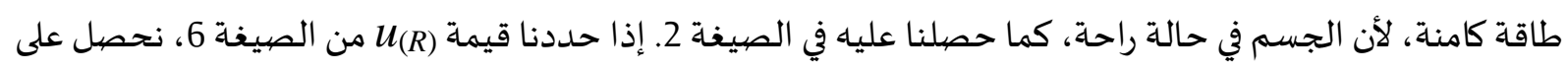

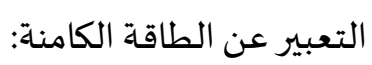

[9] $\quad E_{p}=E_{\alpha=0}=m_{0}\left(\frac{G^{2} M^{2}}{c^{2} R^{2}}-\frac{G M}{R}\right)$

يمكن أيضا كتابة الطاقة الكامنة، باستخدام الصيغة 7، على النحو التالي:

[10]

$$
E_{p}=m_{0} \frac{G M}{R}\left(\frac{R_{0}}{R}-1\right)
$$

إذا قمنا بتحليل معادلة الطاقة الكامنة E $E_{2}$ فيبدو أن لها دائما قيمة سلبية (بإفتراض R R R R ). كذلك نحصل على ان الطاقة الكامنة تساوي الصفر في مكانين، عندما تكون R مساوية لـ

$$
R_{s}=2 \frac{G M}{c^{2}}=2 R_{0}
$$

$$
\text { وبذلك فإن هذا هو نصف قطر شفارتزشيلد الذي يمثل أفق الحدث [10] [11]. }
$$

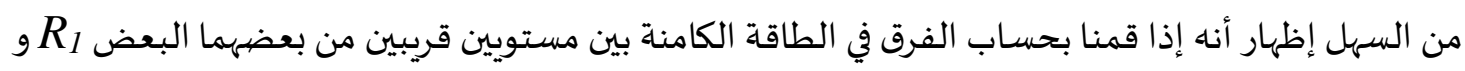
R2 $R_{2}$

$$
\Delta E_{p} \approx m_{0} \underbrace{\frac{G M}{R^{2}}}_{g} \underbrace{\left(R_{2}-R_{1}\right)}_{h}=m_{0} g h
$$

3- م- الطاقة الحركية

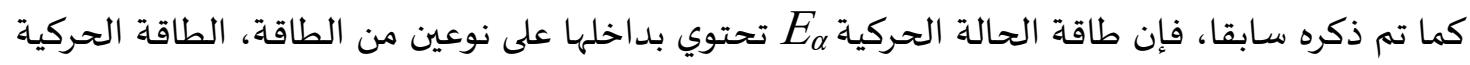

$$
E_{\alpha}=E_{p}+E_{k}
$$

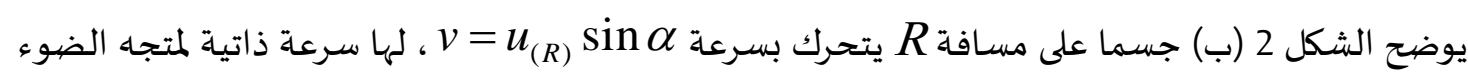
U. U. $\boldsymbol{U}_{(R)}$

$$
E_{\alpha}=m_{0} \boldsymbol{U}_{(R)}\left(\boldsymbol{U}_{(R)}-\boldsymbol{C}\right)=m_{0} u_{(R)}^{2}-m_{0} c u_{(R)} \cos \alpha
$$


عندما نقوم بطرح قيمة الطاقة الكامنة (الصيغة 10) من الطاقة الحركية للحالة، نحصل على القيمة الدقيقة

للطاقة الحركية:

$$
E_{k}=m_{0} c u_{(R)}(1-\cos \alpha)=2 m_{0} c u_{(R)} \sin ^{2}\left(\frac{\alpha}{2}\right)
$$

في مجال جاذبية ضعيف وبسرعات منخفضة،، باستخدام تقديرات تقريبية $\sin ^{2}\left(\frac{\alpha}{2}\right) \approx\left(\frac{\alpha}{2}\right)^{2} \approx \frac{\sin ^{2} \alpha}{4}$

$$
E_{k}=\frac{m_{0} v^{2}}{2}: \text { نحصل على الصيغة المعروفة للطاقة الحركية } u_{(R)}=c
$$

4- - باقة الإفلات وسرعة الإفلات

لكي نتحرر من تأثير مجال الجاذبية، يجب أن نعطي الجسم الصغير الإفات طاقة إفلات، وهي طاقة حركية مساوية في

قيمتها المطلقة للطاقة الكامنة التي حسبناها سابقا، ولكن مع الإشارة المعاكسة، بحيث مجموعها (الطاقة الحركية للحالة)

$$
\text { يساوي الصفر }
$$

[14]

$$
E_{k \text { (Escape) }}=-E_{p}=m_{0}\left(\frac{G M}{R}-\frac{G^{2} M^{2}}{c^{2} R^{2}}\right)=m_{0} \frac{G M}{R}\left(1-\frac{R_{0}}{R}\right)
$$

Rا $R_{0}$ هو الأفق الصفري، وله قيمة صغيرة نسبيا موجودة خارج الكتلة في حالات الثقوب السوداء فقط. لذلك نكانك عندما تكون R أكبر بكثير من ما Rو، يمكن كتابتها تقريبا:

$$
E_{k(\text { Escape })}=m_{0} \frac{G M}{R}
$$

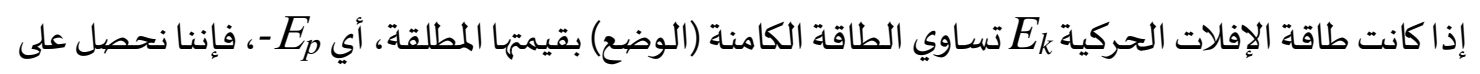

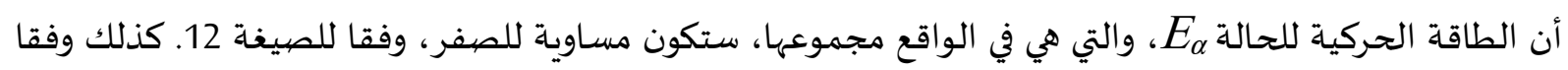
للصيغة 2، نرى أن الطاقة الحركية للحالة هي حاصل الضرب (جداء) النقطي (dot product) لمتجهيين: (2) [2] [2] تصبح $\boldsymbol{V}_{a x}$ هي سرعة الفصل في الزمكان لجسمين قيمة الطاقة الحركية للحالة صفرا عندما تكون الزاوية بين المتجهين

الشكل 3.

$$
\begin{gathered}
\text { لذلك من الصيغتين: } \\
\cos \alpha=\frac{u_{(R)}}{c}=1-\frac{G M}{c^{2} R} \\
v_{(R) \text { (Escape) }}=u_{(R)} \sin \alpha
\end{gathered}
$$

يتم الحصول على سرعة الإفلات:

[16]

$$
v_{(R) \text { (Escape) }} \geq\left(1-\frac{G M}{c^{2} R}\right) \sqrt{2 \frac{G M}{R}-\frac{G^{2} M^{2}}{c^{2} R^{2}}}
$$




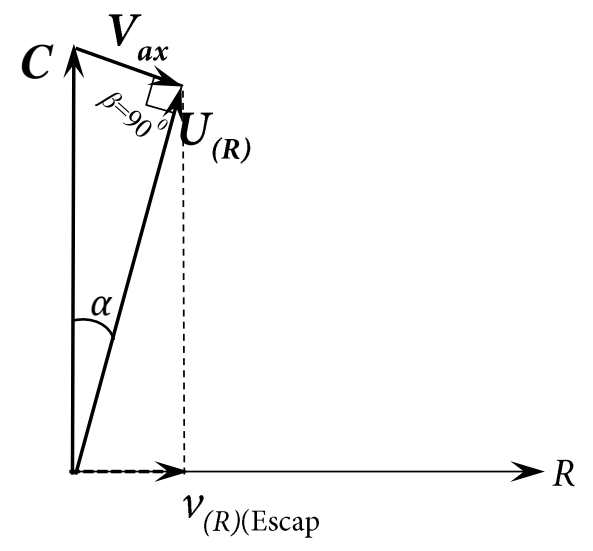

الشكل (3) وصف لسرعة الإفلات في الزمكان، عندما تكون الطاقة الحركية للحالة تساوي الصفر.

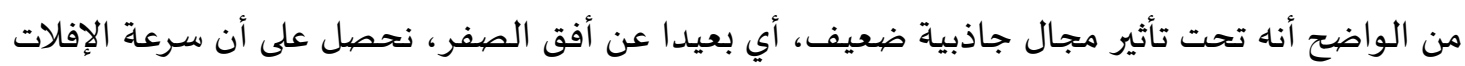

$$
\cdot v_{(R) \text { (Escape) }} \geq \sqrt{2 \frac{G M}{R}}
$$

كما ذكرنا سابقا، يتسبب الجسم ذو سرعة الإفلات في أن تصبح الطاقة الحركية للحالة صفرا، لكل قيمة من R. أي، في الطريق إلى الإفلات، ستظل الزاوية بين المتجهين (R)

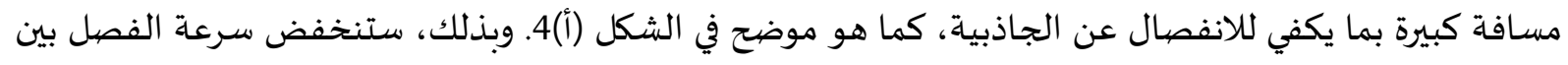

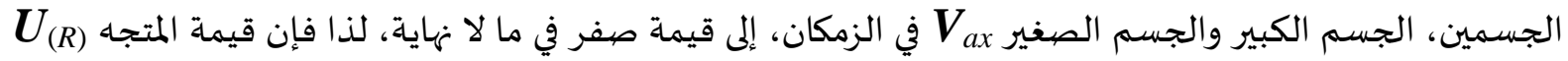
ستكون مساوية لسرعة الضوء Cقيمته المطلقة. شيء آخر يتم الحصول عليه من حقيقة أن الطاقة الحركية للحالة

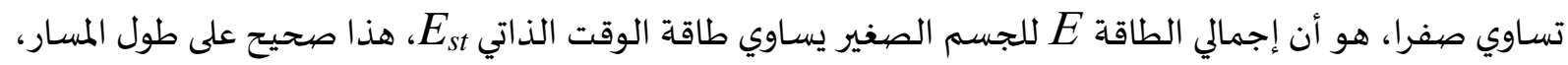
حتى الإفلات، أي في الحالة المعاكسـة، أي عندما ينجذب جسم صغير إلى جسم كبير من مسافة كبيرة من حالة السكون، فإنها

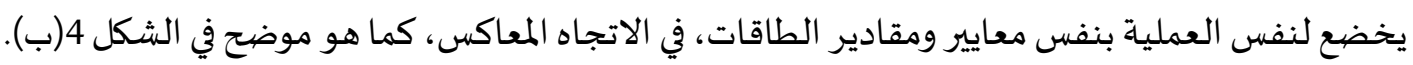

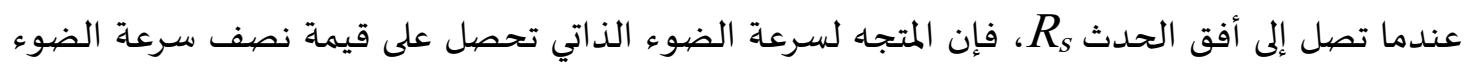
a قيمتها، مساوية ل:

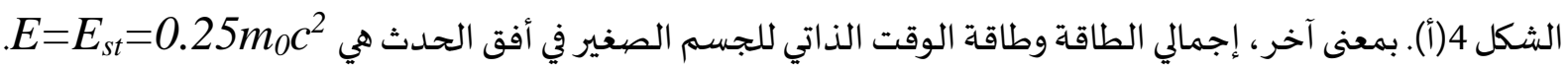
الطاقة الحركية للحالة تساوي صفرا 0 الت من أجل تحرير الجسم الصغير من أفق الحدث، يجب أن نستثمر فيه طاقة حركية تساوي 


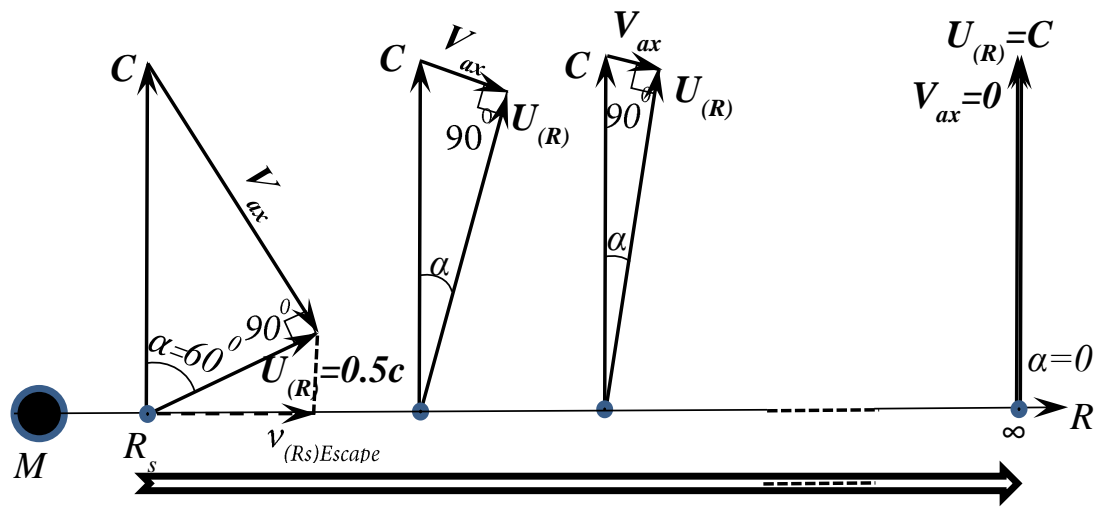

(i)

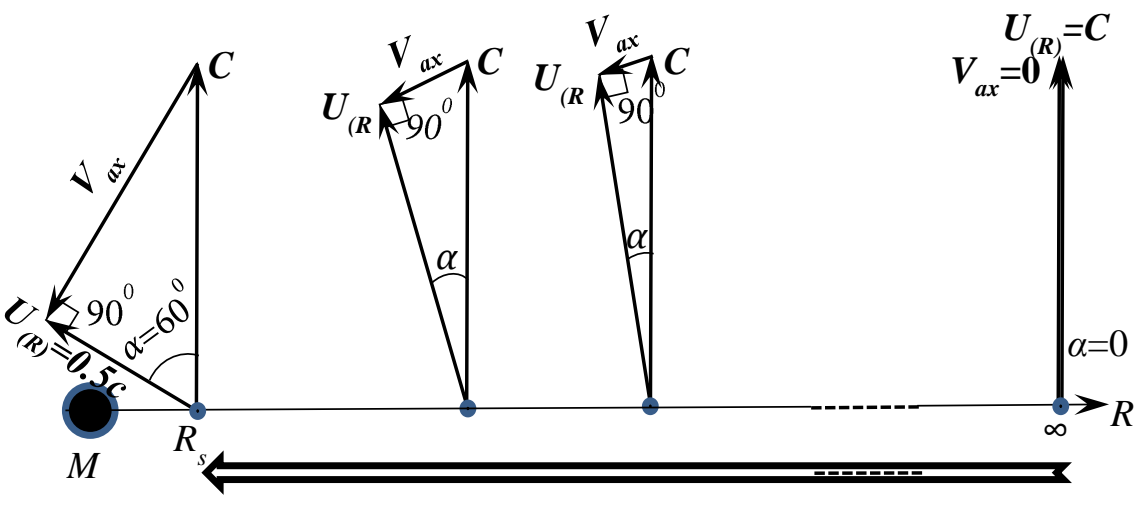

$(+)$

الشكل (4) (أ) يحافظ الجسم ذو سرعة الإفلات على الطاقة الحركية للحالة كصفرعلى طول الطريق حتى ينفصل عن تأثير مجال الجاذبية.

(ب) العملية قابلة للعكس، أي أن الجسم الذي يقترب من مجال الجاذبية يتلقى نفس قيم الطاقات والسرعات.

5- ت توقيت القمر الصناعي

كما هو معروف جيدا، يجب مزامنة أقمار GPS الصناعية في الوقت المناسب على الأرض للحصول على أقصى

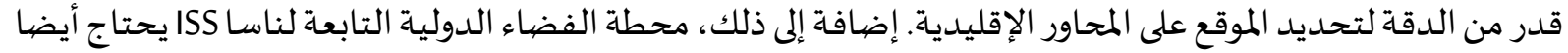

إلى المزامنة مع ساعة على الأرض لإجراء تجارب معينة. لذلك، نأخذ هذين المثالين كمثال لاختبار نموذجنا [12]. بالنظر إلى الأرض، وهي كوكب صغير نسبيا، نحاول اختبار الصيغة 6، نحصل على فرق صغير جدا، بين المتجاء لسرعة الضوء الذاتي C لجسمى حر (خارج مجال الجاذبية)، والذي له قيمة مطلقة لـ سرعة الضوء تحت تأثير مجال الجاذبية للأرض، بحيث يمكن أن يصل إلى قيمة قصوى، على سطح الأرض، بمعدل فأ فرقا زمنيا قدره الأرض أبطأ بمقدار 60 ميكروثانية في اليوم، نسبة إلى الجسم الحر، كما هو موضح في الشكل 5. 
الفرق الزمني بين جسم حر (في هذه الحالة نأخذ مركز الأرض كمرجع، كنقطة كتلة حرة في الفضاء) وجسم تحت

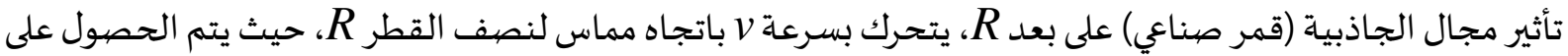
الزاوية الطاقة $\alpha$ من التعبير $\alpha=u_{(R)} \sin v$ ، نحسب إسقاط المحور الزمني، كما هو موضح في الشكل 5: $c \Delta T=c T-u_{(R)} T \cos \alpha$

$$
\Delta T=T(1-\cos \alpha+\underbrace{\frac{G M}{c^{2} R}}_{\frac{R_{0}}{R}} \cos \alpha)
$$

تعبر الصيغة 17 عن الفروق الزمنية بين إطارين مرجعيين لجسمين، أحدهما جسم ثابت حر (بدون تأثير

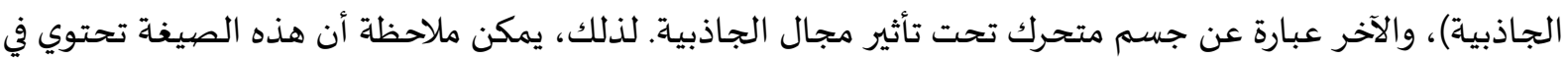

داخلها على نظريتي النسبية الخاصة والعامة معا.

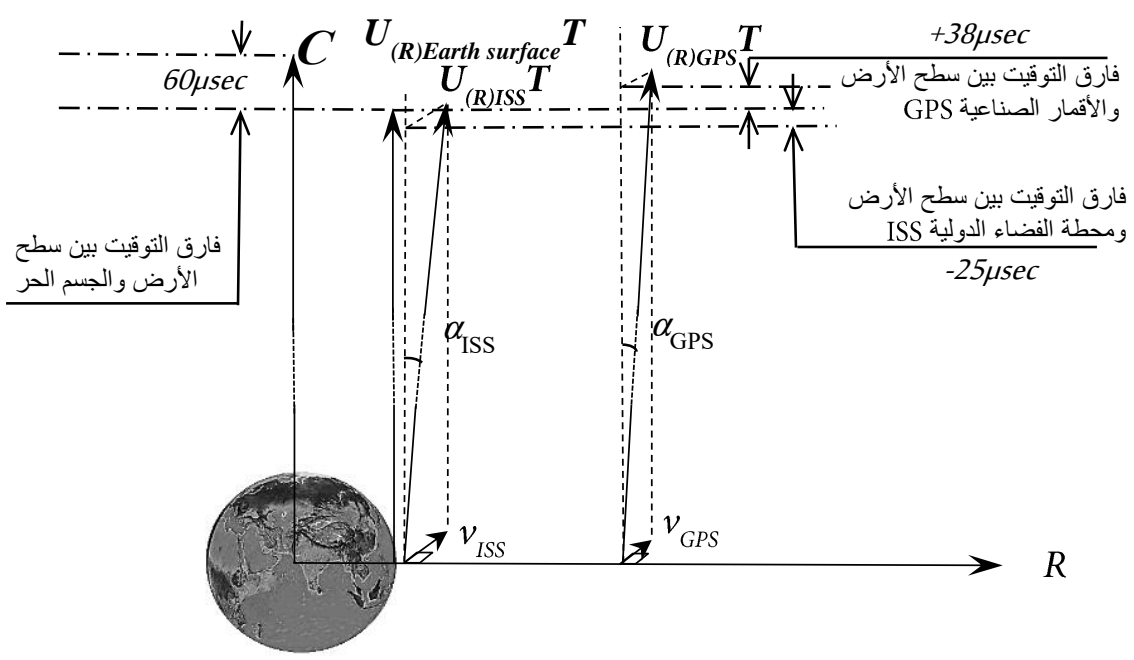

الشكل (5) وصف الفروق الزمنية في اليوم، بين الأقمار الصناعية ISS وGPS والوقت على الأرض. تم إنشاء جدول لحساب فروق التوقيت في اليوم، وفقا للصيغة 17 والبيانات:

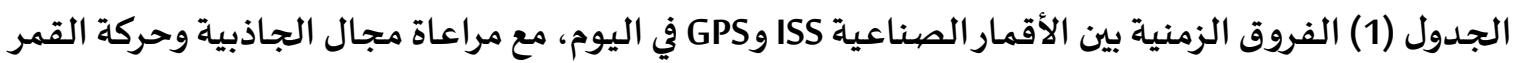
الصناعي.

\begin{tabular}{|c|c|c|c|c|c|}
\hline & $\begin{array}{c}\text { المسافة } \\
R \\
(\mathrm{~m})\end{array}$ & $\begin{array}{c}\text { سرعة } \\
V \\
(\mathrm{~m} / \mathrm{sec})\end{array}$ & $\begin{array}{c}\text { الزاوية الطاقة } \\
\boldsymbol{\alpha} \\
(\mathrm{rad})\end{array}$ & 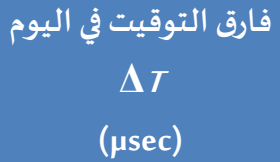 & $\begin{array}{c}\text { فالنسبة لسطح الأرقيت فيوض } \\
\text { (رsec) }\end{array}$ \\
\hline الجسم الحر (مركز الأرض) & $\mathbf{0}$ & $\mathbf{0}$ & $\mathbf{0}$ & $\mathbf{0}$ & 60 \\
\hline على سطح الأرض & 6357000 & $\mathbf{0}$ & $\mathbf{0}$ & 60 & $\mathbf{0}$ \\
\hline القمر الصناعي ISS & 6767000 & 7700 & $2.56838+10^{-5}$ & $85 \mu \mathrm{sec}$ & $-25 \mu \mathrm{sec}$ \\
\hline $\begin{array}{c}\text { الأقمار الصناعية } \\
\text { GPS }\end{array}$ & 26541000 & 3874 & $1.29219+10^{-5}$ & $22 \mu \mathrm{sec}$ & $+38 \mu \mathrm{sec}$ \\
\hline
\end{tabular}


يمكن ملاحظة نتائج الجدول 1 بشكل تخطيطي في الشكل 5، الذي يصور السرعة الذاتية لمتجهات الضوء لكل

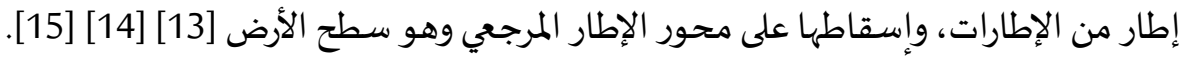

\section{6- سرعة الضوء تحت تأثير مجال الجاذبية}

يوضح الشكل 6 (أ) المتجاه لسرعة الضيوء الذاتي لجسم صغير لهير يبتعد عن مركز الجسم الكبير (النجم)، عند

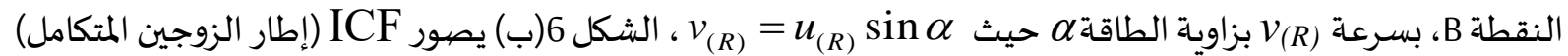
[1] [2] [3]. يوضح الشكل 6 (ج) خط الضوء من النقطة B إلى النقطة A. في الزمكان، يتم اختيار نقطتين قريبتين جدا،

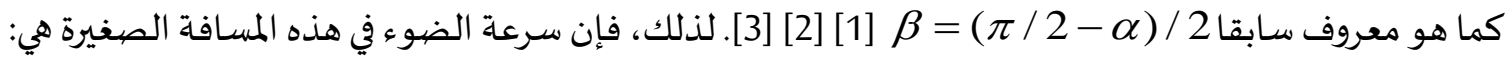

$$
\text { Gravitational light speed }=\frac{R_{A}}{t_{A}}=\frac{u_{(R)} t_{A}}{t_{A}}=u_{(R)}=c-\frac{G M}{c R}
$$

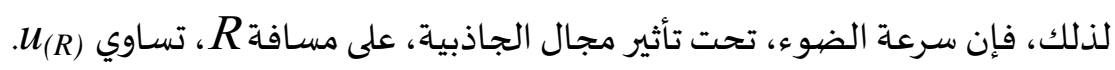

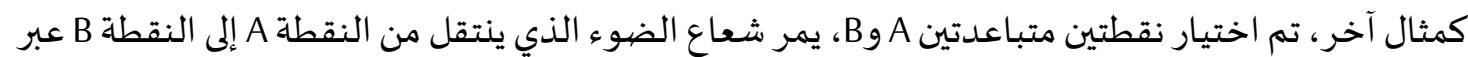

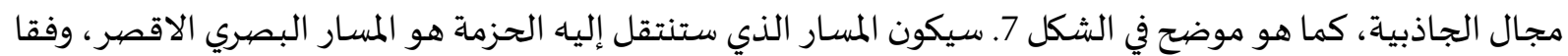

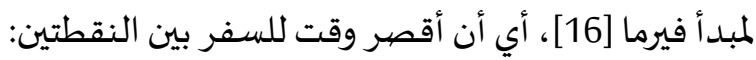

[19]

$$
T_{[\min ]}=\int_{A}^{B} \frac{|\mathrm{d} s|}{u_{(R)}}
$$

كما يتضح من الشكل 7 فإن مسار شعاع الضيوء الثقالي ليس مستقيما، ويعتمد على حجم مجال الجاذبية الذي أنداء

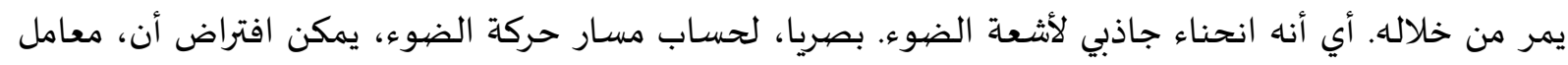

$$
\text { الانكسار للضيوء، عند المستوى R }
$$

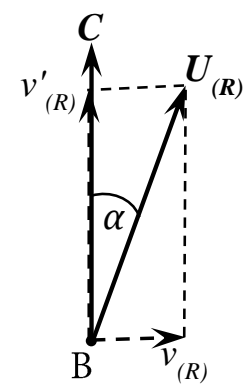

(a)

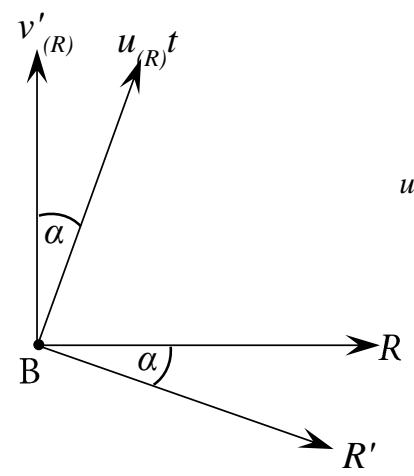

(b)

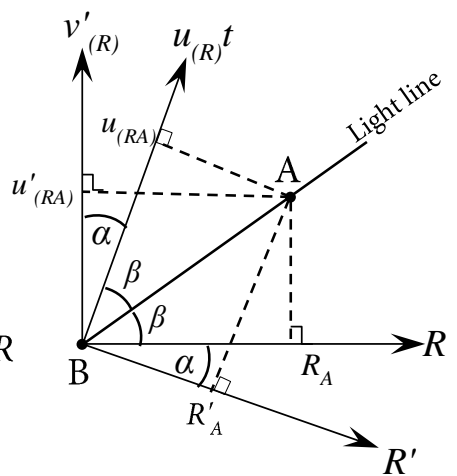

(c)

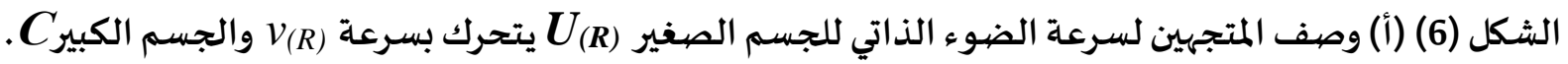
ICF (ب) (إطار الزوجين المتكامل).

(ج) وصف مسار الضيوء في إطار ICF. 

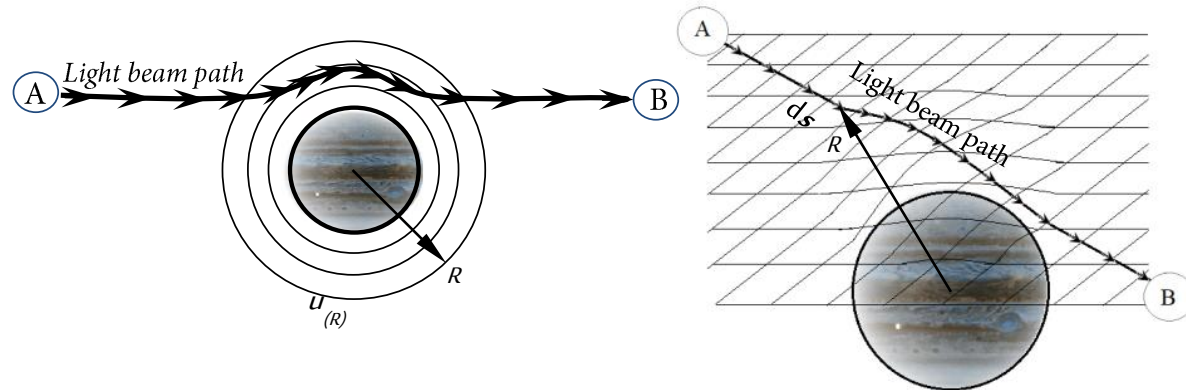

الشكل (7) وصف مسارشعاع من الضيوء يتحرك من النقطة A إلى النقطة B ، عبر مجال الجـاذبية.

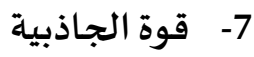

يمكن القول إن مجال الجاذبية يخلق مستويات مختلفة من الطاقة، والتي تعتمد بشكل مباشر على المسسافة

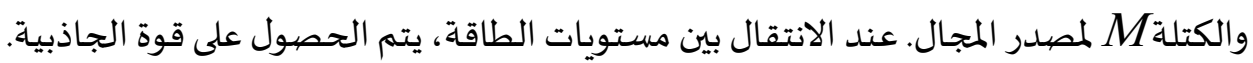
تؤثر قوة الجاذبية على جسم صغير في حالة السكون تحت تأثير مجال الجاذبية لجسم كبير M. R، تسمى وزن الجسم، وتساوي التدرج للطاقة الكامنة:

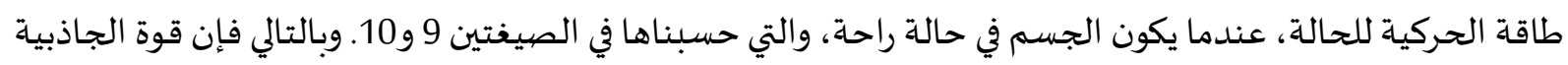

$$
\boldsymbol{F}_{(R)}=-\frac{\mathrm{d} E_{p}}{\mathrm{~d} R}=-m_{0}\left(\frac{G M}{R^{2}}-2 \frac{G^{2} M^{2}}{c^{2} R^{3}}\right)
$$

$$
\boldsymbol{F}_{(R)}=-m_{0} \frac{G M}{R^{2}}\left(1-\frac{2 R_{0}}{R}\right)
$$

يمكن أيضا كتابة الصيغة 20 على النحو التالي:

$$
\text { القيمة }
$$




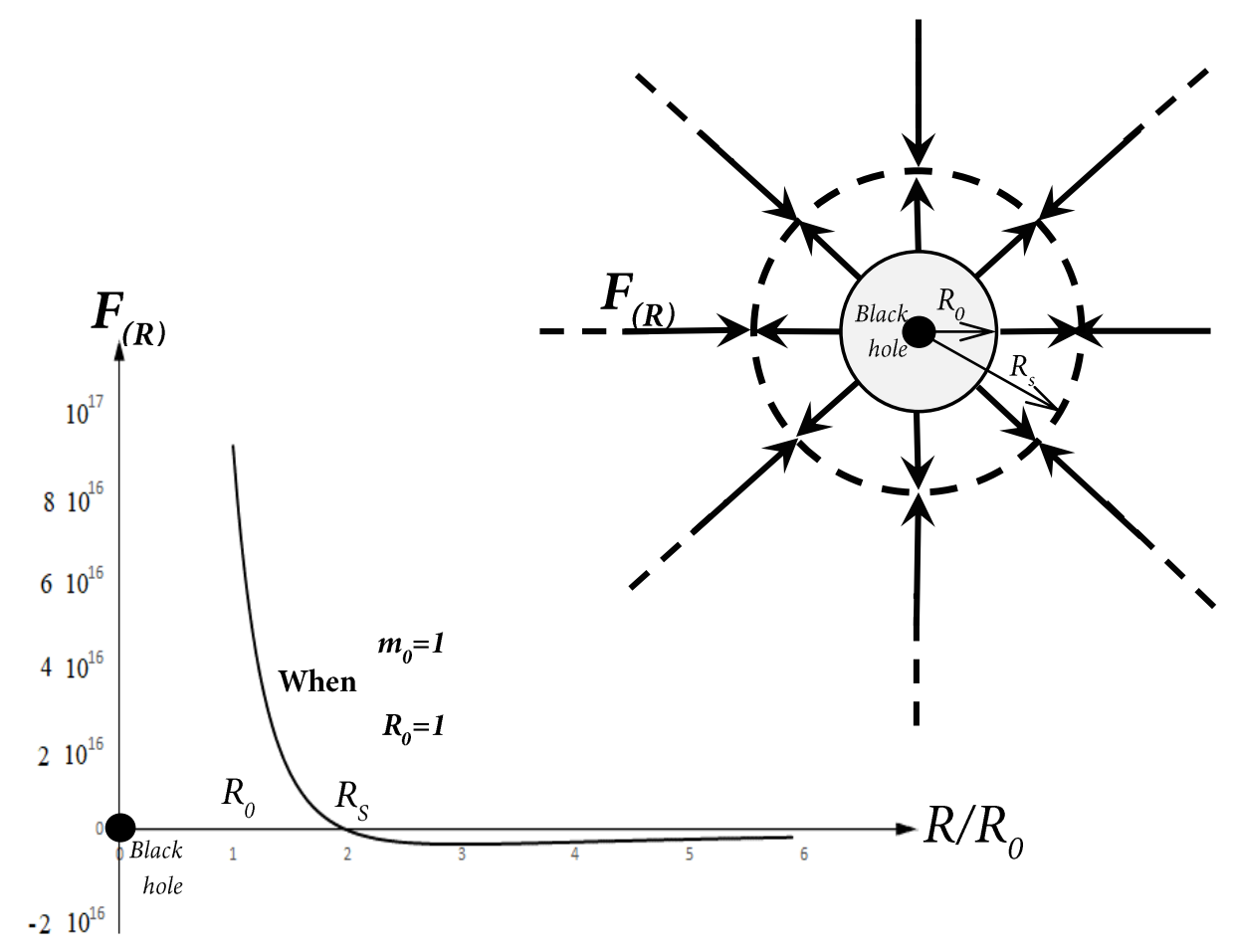

$$
\text { الشكل (8) وصفف اتجاه ومقدار القوة الجـاذبية في الثقب الأسـود. }
$$

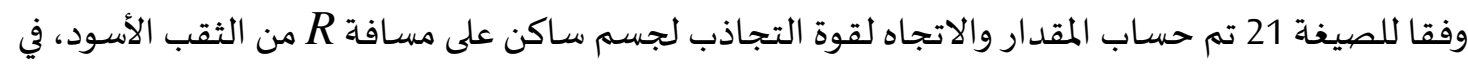

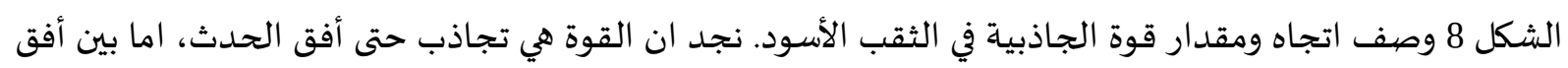

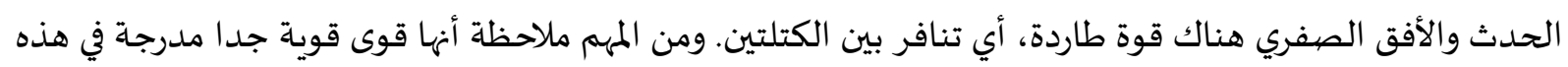

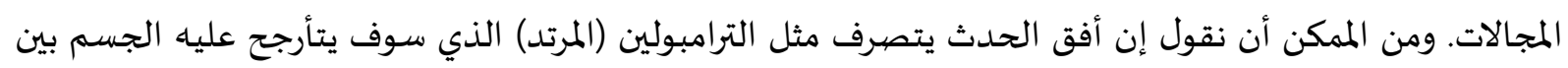

$$
\text { تججاذب وتنافر. }
$$

على سبيل المثال، الجسم الذي يستقر في هاية العملية في أفق الحدث تكون طاقته محافظة، وبالتالي فرق الطاقة، وهو

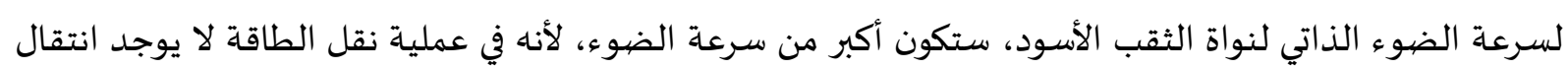

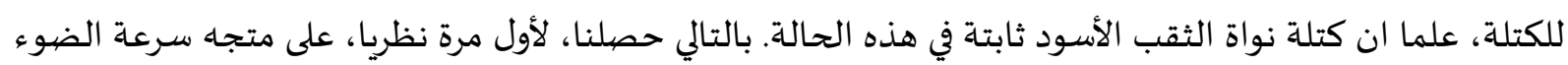

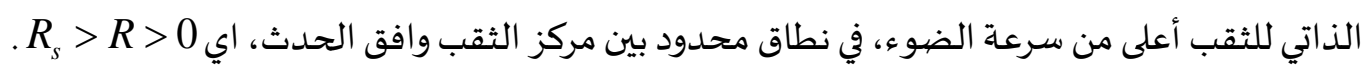

8- المناقشـة والخاتمة.

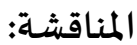

نموذج فيزيائي جديد، هو محصلة هذا البحث، ليس من خلال المسافة الزمكانية، السرعة، التسارع والزخم، كما

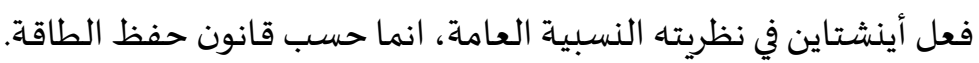

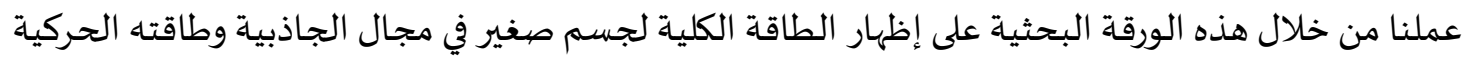

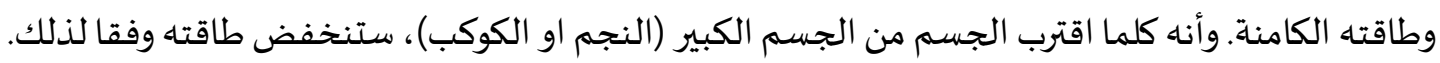


قمنا أيضا بحسـاب وشرح الأفق الصفري، والفرق الزمني بين جسمين، والكتلة الفعالة للجسم، وقوة الجاذبية

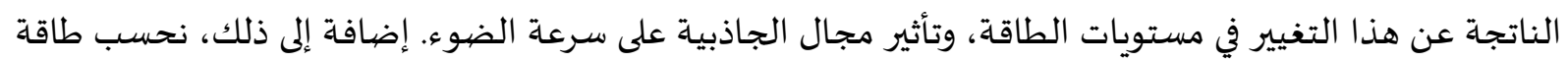
الإفلات وسرعة الإفلات، مما يدل على أن الجسم الذي يتمتع بسرعة الإفلات يحافظ على الطاقة الحركية للحالة على أهنا

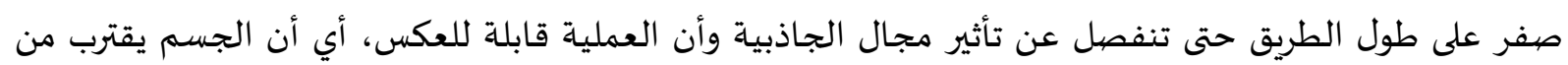

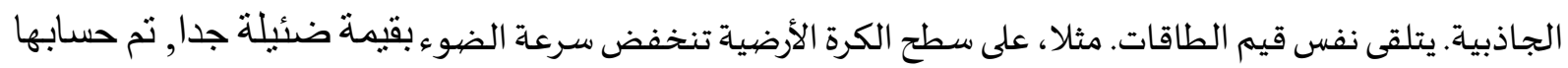

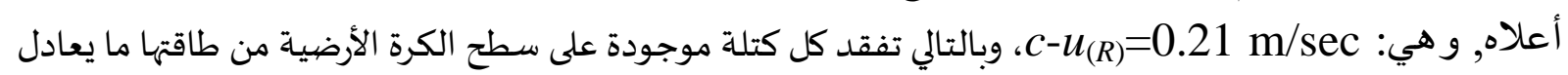

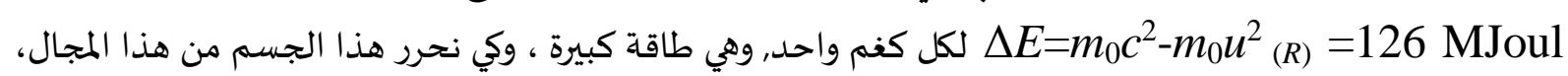
علينا ان نبذل طاقة بقيمة نصف تلك الطاقة، أي

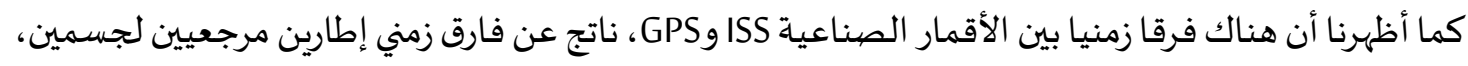

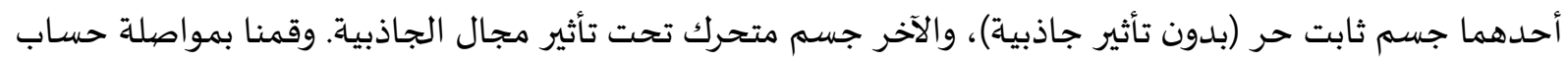
سرعة الضيوء، وتبيين تأثير مجال الجاذبية عليه. في الأخير قمنا بتوضيح أن مجال الجاذبياة يخلقير مستويات طاقة مختلفة، والتي تعتمد بشكل مباشر على المسافة R والكتلة M لمصدر المجال. في الانتقال بين مستويات الطاقة، يتم الحصيول على قوة الجاذبية.

الخاتمة.

يقدم هذا النموذج قوة الجاذبية بطريقة مختلفة ومبتكرة، مما يعطي إجابات لقضايا تتعلق بالعديد من

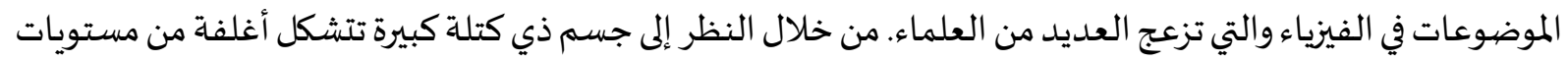
الطاقة، فيوضح هذا النموذج أن مستويات الطاقة هذه هي السبب في تكوين قوة الجاذبية.

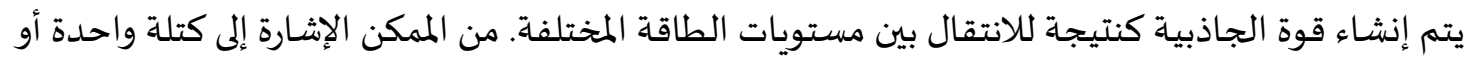

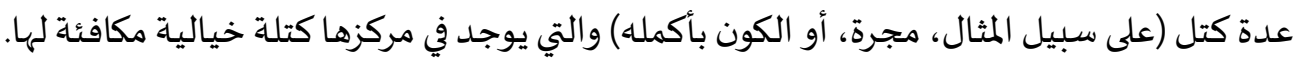

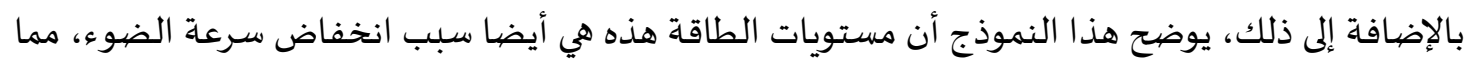

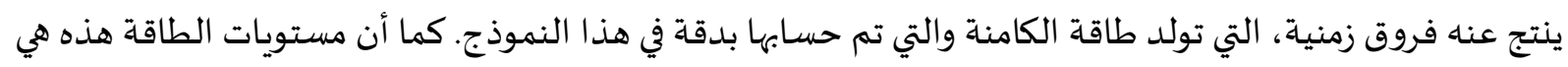
السبب في انحناء حركة الضوء التي تمر عبر مجال الجاذبية.

الدراسات المستقبلية

تعتبر هذه الورقة البحثية بوابة لأوراق بحثية أخرى، والتي سوف ندرس فهها مواضيع إضافية، بما في ذلك: 1- ما الذي تعتمد عليه قوة الجاذبية، عندما تكون هناك ظروف أولية مختلفة، على سبيل المثال السرعة الابتدائية،

أو الكتلة السالبة، أي جسم صغير الدير بزاوية طاقة كبيرة.

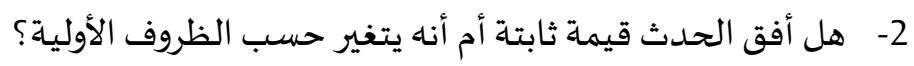

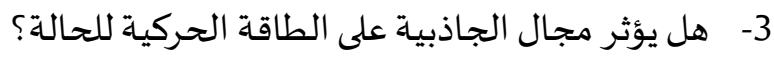

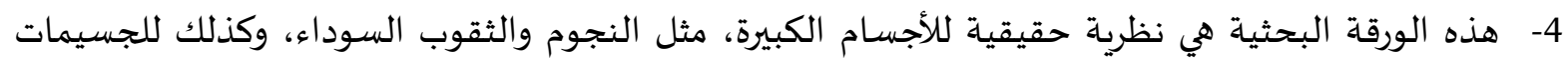

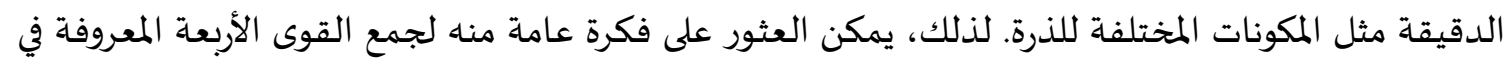

الفيزياء المرجع [17].

قائمة مختصرات ورموز 


\begin{tabular}{|c|c|c|}
\hline هو المتجه ذوسرعة الضيوء، بقيمته المطلقة، و اتجاهه محور الزمن، في الأطر القصورية. & الذاتجه للسرعة الضيوء & C \\
\hline هو المتجه ذوسرعة اقل من سرعة الضيوء (بسبب مجال الجاذبية)، بقيمته المطلقة، & الذاتي للجسم ل الصغرعة الضيوء & $U_{(R)}$ \\
\hline 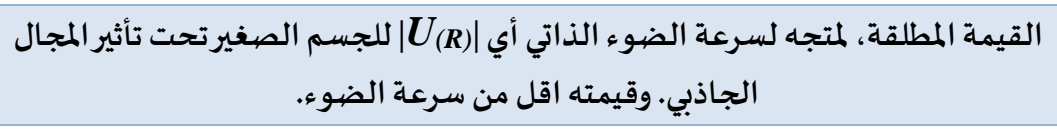 & قيمة المتجه لسرعة الضيوء & $u_{(R)}$ \\
\hline الزاوية التي تفصل بين المتجهين لسرعة الضهوء الذاتي C و & 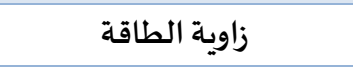 & $\alpha$ \\
\hline 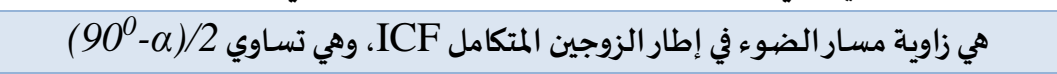 & 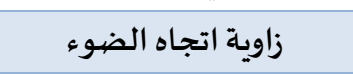 & $\beta$ \\
\hline 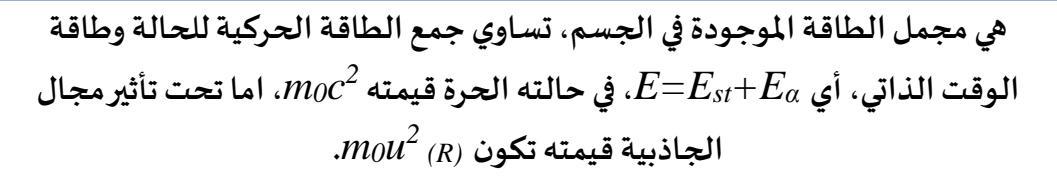 & الطاقة الكلية الية & $\boldsymbol{E}$ \\
\hline 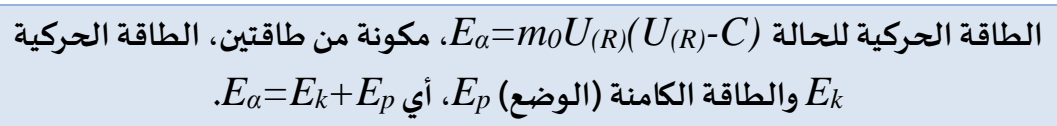 & الطاقة الحركية للحالة & $\boldsymbol{E}_{\boldsymbol{a}}$ \\
\hline طاقة الوقت الذاتي & طاقة الوقت الذاتي للجسم & $\boldsymbol{E}_{\text {st }}$ \\
\hline 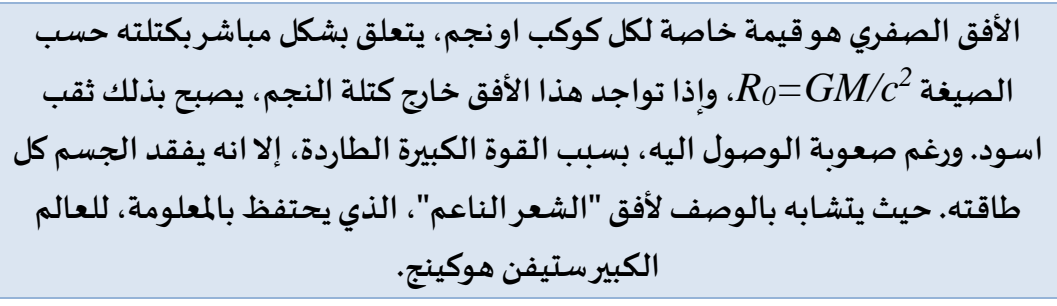 & الأفق الصفري & $R_{0}$ \\
\hline أفق الحدث اونصف قطرسفارتزشيلد يساوي كونه أفق يفصل بين قوة التجاذب والقوة الطاردة. & أفق الحدث & $R_{s}$ \\
\hline 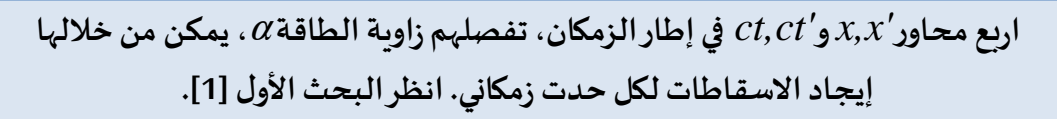 & 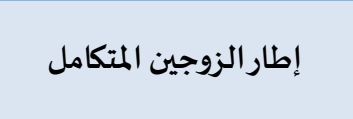 & ICF \\
\hline
\end{tabular}

قائمة المراجع.

[1] Tannous, E. (Sep. 2021), Negative Mass, Arab Journal for Science and Research Publishing (AJSRP), DOI: 10.26389/AJSRP.D210421.

DOI: AJSRP إدوار طنوس, (سبتمبر 2021), الكتلة السالبة, المجلة العبية للعلوم ونشر الأبحاث 10.26389/AJSRP.D210421

[2] Tannous, E. (2019), Negative Mass, Journal of Modern Physics, 10, 861-880. https://doi.org/10.4236/jmp.2019.107057

[3] Tannous, E. (2021), Energetic Angle, Newest Updates in Physical Science Research, 1, 45-64. https://doi.org/10.9734/bpi/nupsr/v1/7088D

[4] O'Connor, J.J. and Robertson, E.F. (1996) General Relativity. History Topics: Mathematical Physics Index, School of Mathematics and Statistics, Scotland.

[5] Feynman, R. (1970) The Feynman Lectures on Physics. Pearson P T R, 1st Edition, Volume 1, Chapter 4. https://www.feynmanlectures.caltech.edu/I_04.html 
[6] Newton, I. (1966) Philosophiae Naturalis Principia Mathematica (Mathematical Principles of Natural Philosophy, 1687). Translated in English by A. Motte, Revised and Annotated by F. Cajori (University of California Press). https://doi.org/10.5479/sil.52126.39088015628399

[7] Wald, R.M. (1997) Gravitational Collapse and Cosmic Censorship. Black Holes, Gravitational Radiation and the Universe. Springer, Dordrecht, 69-86.

[8] Günther, H. and Müller, V. (2019) Einstein's Energy-Mass Equivalence. The Special Theory of Relativity: Einstein's World in New Axiomatics. Springer, Singapore, 97-105. https://doi.org/10.1007/978-981-13-7783-9_7

[9] Overbye, D. (2015) Black Hole Hunters. NASA. Archived from the Original.

[10] Hamilton, A. (2020) Journey into a Schwarzschild Black Hole, JILA: A Joint Institute of NIST and the University of Colorado Boulder. https://jila.colorado.edu/ ajsh/insidebh/schw.html

[11] Thorne, K.S. and Hawking, S. (1994) Black Holes and Time Warps: Einstein's Outrageous Legacy. W. W. Norton \& Company, New York, 134-135.

[12] Narayankar, S. (2019) International Journal of Advanced Research (IJAR), 7, 782-789.

[13] Hassani, Sadri. (2011) From Atoms to Galaxies: A Conceptual Physics Approach to Scientific Awareness. CRC Press. p. 433. ISBN978-1-4398-0850-4. Extract of page 433 https://doi.org/10.21474/IJAR01/8697

[14] Topper, David. (2012) How Einstein Created Relativity out of Physics and Astronomy(illustrated ed.). Springer Science \& Business Media. p. 118. ISBN 978-1-4614-4781-8.Extract of page 118.

[15] Kogut, John B. (2012) Introduction to Relativity: For Physicists and Astronomers(illustrated ed.). Academic Press. p. 112. ISBN 978-0-08-092408-3.

[16] Born, M. and Wolf, E. (1975) Principles of Optics. Cambridge University Press, Cambridge, p. 740.

[17] Davies, P. (1986) The Forces of Nature. 2nd Edition, Cambridge University Press, Cambridge. 